\title{
Upregulation of MAPK10, TUBB2B and RASL11B may contribute to the development of neuroblastoma
}

\author{
JIANGTAO LIU ${ }^{1,2}$ and YULIN LI ${ }^{1}$ \\ ${ }^{1}$ The Key Laboratory of Pathobiology, Ministry of Education, Department of Pathobiology, \\ College of Basic Medical Sciences, Jilin University; ${ }^{2}$ The Department of Pediatric Clinic, \\ The First Hospital of Jilin University, Chuangchun, Jilin 130000, P.R. China
}

Received March 16, 2018; Accepted November 7, 2018

DOI: $10.3892 / \mathrm{mmr} .2019 .10589$

\begin{abstract}
The present study aimed to investigate genes and transcription factors (TFs) that may contribute to neuroblastoma (NB) development. The GSE78061 dataset that included 25 human NB cell lines and four retinal pigment epithelial cell lines was used to analyze differentially expressed genes (DEGs) between groups. Functional enrichment analysis and protein-protein interaction (PPI) network analysis were performed for the identified DEGs. Additionally, submodule analysis and TF-target regulatory networks were conducted. The relative mRNA expression levels of mitogen-activated protein kinase 10 (MAPK10), tubulin $\beta 2 \mathrm{~B}$ class IIb (TUBB2B), RAS like family 11 member B (RASL11B) and integrin subunit $\alpha 2$ (ITGA2) in the NB cell line SH-SY5Y were compared with retinal pigment epithelial cell lines. A set of 386 upregulated and 542 downregulated DEGs were obtained. Upregulated DEGs were significantly associated with the 'neuron migration' and 'dopaminergic synapse signaling' pathways, whereas, downregulated DEGs were primarily involved in 'focal adhesion' such as ITGA2 and ITGA3. In the PPI networks analyzed, MAPK10, dopa decarboxylase (DDC), G protein subunit $\gamma 2$ (GNG2), paired like homeobox 2B (PHOX2B), TUBB2B, RASL11B, and ITGA2 were hub genes with high connectivity degrees. Additionally, PHOX2B was predicted to be a TF regulating TUBB2B in the regulatory network. The expressions of MAPK10, TUBB2B, RASL11B and ITGA2 were detected by reverse transcription-quantitative polymerase chain reaction in the NB cell line SH-SY5Y, and were consistent with the present bioinformatics results, suggesting that MAPK10, DDC, GNG2, PHOX2B, TUBB2B,
\end{abstract}

Correspondence to: Dr Yulin Li, The Key Laboratory of Pathobiology, Ministry of Education, Department of Pathobiology, College of Basic Medical Sciences, Jilin University, 126 Xinmin Avenue, Chuangchun, Jilin 130000, P.R. China

E-mail: ylli2889@hotmail.com

Key words: neuroblastoma, differentially expressed genes, protein-protein interaction network, transcription factor, regulatory network
RASL11B, ITGA2 and ITGA3 may contribute to NB development. Additionally, the present study identified a novel significant association between the increased expression levels of MAPK10, TUBB2B and RASL11B, and NB cells.

\section{Introduction}

Neuroblastoma (NB) is an embryological malignant disease primarily affecting the adrenal medulla; however, NBs may occur in the abdomen, chest, pelvis and other areas where nerves terminate (1). NB is among the most common types of pediatric solid tumors, and $\sim 90 \%$ of NB cases occur in children $<5$ years of age with a median age of 18 months (2). NB accounts for $\sim 8 \%$ of total pediatric malignancies and $\sim 15 \%$ of total cancer mortalities (3). The International Neuroblastoma Staging System defined four stages for NB (4), and the survival rate is determined by the age and the disease stage of patients with NB (3). Although multimodal therapies have been developed to improve the survival rate of patients with $\mathrm{NB}$, the overall survival rate remains $<40 \%$, and patients with NB at stage four have a five-year survival rate of $30-35 \%$ (5). Therefore, early diagnosis and pathogenesis of NB are important factors for improving the survival rate of patients with NB.

A number of potential markers for early diagnosis and therapeutic targets of NB have been investigated. The neuropeptide Y (NPY), a sympathetic neurotransmitter expressed in the central nervous system, was significantly overexpressed in NB tumor tissues of a TH-MYCN mouse model and its increased expression level was associated with poor survival, tumor metastasis and relapse of NB (6). Astrocyte elevated gene-1, which encodes for metadherin, was upregulated in NB and promotes NB cell proliferation by activating the phosphatidylinositol-3-kinase/AKT serine/threonine kinase 1 signaling pathway (7). Pim 1 proto-oncogene, serine/threonine kinase (PIM1), PIM2 and PIM3 are proto-oncogenes encoding for Ser/Thr protein kinases; the high expressions of these genes were significantly correlated with poor overall survival of patients with NB (8). Galinski et al (9) suggested a novel therapeutic biomarker, exportin-1 (XPO1), for patients with NB, and demonstrated that high expression of XPO1 in patients with NB was associated with poor outcomes. Additionally, downregulation of 
bone morphogenetic protein receptor 2 increased the cell growth and clonogenicity of NB cells (10). A previous study demonstrated that neuropilin 1 may be a promising diagnostic biomarker for NB, and its knockdown increased the migration and invasion of SK-N-AS NB cells (11). In contrast, knockdown of solute carrier family 39 member 8 inhibited the progression and metastasis of NB (12). Transcription factors (TFs) serve important roles in the initiation and progression of NB. POU class 4 homeobox 2 (POU4F2) TF regulates the growth, invasive capacity and proliferation of NB cells, and decreased expression of POU4F2 may inhibit these effects (13). In 2010, Souzaki et al (14) suggested that increased expression levels of sonic hedgehog, GLI family zinc finger 1 and patched 1 are involved in activating the hedgehog signaling pathway, and it was suggested that activation of this pathway is involved in NB differentiation (14). Although numerous previous studies identified potential markers and examined the mechanisms associated with NB development, the molecular mechanisms underlying NB progression remain unclear.

In the present study, the GSE78061 dataset from Hart et al (15) was downloaded from the Gene Expression Omnibus (GEO) database, to conduct a bioinformatics analysis. The GSE78061 dataset was analyzed to identify differentially expressed genes (DEGs) between NB cell lines and normal retinal pigment epithelial (RPE) cells. Furthermore, the protein-protein interactions (PPIs) of the proteins encoded by DEGs were investigated, in order to examine the TFs regulating the DEGs, and to investigate the molecular mechanisms underlying NB. Additionally, the present study may provide novel diagnostic and therapeutic markers for NB.

\section{Materials and methods}

Microarray data. The GSE78061 dataset (15) used in the present study was downloaded from GEO (www.ncbi.nlm. nih.gov/geo/), based on the Affymetrix human gene 1.0 ST array (Thermo Fisher Scientific, Inc., Waltham, MA, USA). Expression data from 25 human NB cell lines and four RPE cell lines were analyzed in the GSE78061 dataset.

Data preprocessing. Raw expression data in CEL format were downloaded and the Oligo package in $\mathrm{R}$ (version 3.4.1; CRAN.R-project.org/bin/windows/base) was used to read the chip data (16). The expression data of all samples were preprocessed and normalized using the robust multi-array average method (17), which included background-adjusted quantile normalization, log-transformed perfect match values and summarization.

DEGs and hierarchical cluster analyses. The probe levels were extracted from the expression matrix following data preprocessing. The linear models for microarray data (limma; version 3.26.9; www.bioconductor.org/packages/3.2/bioc/html/limma.html) package in $\mathrm{R}$ was applied to screen for DEGs between NB samples and RPE samples (18). The threshold values for identifying DEGs were defined as adj.p.val $<0.01$ and $\log _{2}$ fold-change $(F C) \mid>2$. Additionally, the pheatmap package was used (version 1.0.8; cran.r-project. org/web/packages/pheatmap/index.html) in $\mathrm{R}$ to draw two-dimensional clustering heatmaps based on the expression levels of the identified DEGs.

Functional and pathway enrichment analyses. The Gene Ontology (GO; www.geneontology.org) database provides functional categorization and annotations of biological process (BP), molecular function (MF) and cellular component (CC) for large-scale transcriptomic data (19). The Kyoto Encyclopedia of Genes and Genomes (KEGG; www.genome. $\mathrm{jp} / \mathrm{kegg}$ ) is a comprehensive database that integrates genomic, chemical and systemic information to define the function of genes or proteins involved in various metabolic and regulatory pathways (20). Additionally, the Database for Annotation, Visualization and Integrated Discovery (DAVID; version 6.8; david.abcc.ncifcrf.gov) (21) is a tool for performing GO and KEGG pathway enrichment analyses. In the present study, DAVID was used to perform functional enrichment analysis for upregulated and downregulated genes. The significantly enriched results were selected with the following cutoffs: Enriched gene count $\geq 2$ and $\mathrm{P}$-value $<0.01$.

PPI network and submodule analyses. The Search Tool for the Retrieval of Interacting Genes (STRING; string-db. $\mathrm{org} /$ ) (22) is a database that integrates functional interactions among proteins in numerous organisms. The weighted protein interactions provide information associated with functional linkages between proteins and genomic associations among gene products. In the present study, the STRING database was used to investigate interacting proteins encoded by DEGs and the default value of the combined score $>0.4$ was set as the criteria for selecting significant interactions. Cytoscape software (www.cytoscape.org/; version 3.3.0) is a visualization tool for analyzing molecular networks (23), in which nodes represent proteins, and edges connecting the nodes indicate their associations. Key nodes in the PPI network were obtained based on the ranks of their connectivity degrees.

In the PPI network, the distances between two nodes are associated with their function and nodes tend to cluster together based on similar function. Therefore, submodule analysis is an effective strategy for predicting protein function. The Molecular Complex Detection (MCODE, version 1.4.2) tool was used to perform submodule analysis for the PPI network (24).

TF prediction and construction of transcriptional regulation network. Transcriptional Regulatory Relationships Unravelled by Sentence-based Text-mining (TRRUST; version 2; www. grnpedia.org/trrust) is a database that contains 8,444 and 6,552 TF-target regulatory associations of 800 human TFs and 828 mouse TFs, respectively. The TRRUST database draws from 11,237 PubMed articles and represents small-scale experimental studies of transcriptional regulation (25). The TRRUST database was used to identify the TFs among the DEGs and the regulatory associations between the TFs and their target genes. Subsequently, the predicted target genes among the DEGs were screened, and a transcriptional regulation network was constructed using Cytoscape software.

Validation of gene expression by reverse transcriptionquantitative polymerase chain reaction ( $R T-q P C R)$. RT-qPCR 
analysis was performed to detect the gene expression levels of numerous DEGs predicted to be associated with human NB. hTERT RPE-1 cells (Jennio Bioech Co., Ltd., Guangzhou, China) were cultured in RPMI-1640 medium (Gibco; Thermo Fisher Scientific, Inc.), and SH-SY5Y cells (cat. no. SCSP-5014; Stem Cell Bank, Chinese Academy of Sciences, Shanghai, China) were cultured in Dulbecco's modified Eagle's medium/nutrient mixture F-12 (Gibco; Thermo Fisher Scientific, Inc). Both cells lines were cultured at $37^{\circ} \mathrm{C}$ under a humidified $5 \% \mathrm{CO}_{2}$ atmosphere. Total RNA was extracted using TRIzol ${ }^{\circledR}$ reagent (Invitrogen; Thermo Fisher Scientific, Inc.), and RNA was reverse-transcribed using PrimeScript RT master mix (Takara Bio, Inc., Otsu, Japan) for cDNA synthesis at $37^{\circ} \mathrm{C}$ for $60 \mathrm{~min}$, followed by $37^{\circ} \mathrm{C}$ for $5 \mathrm{~s}$. Following cDNA synthesis, qPCR was conducted using Power SYBR Green PCR master mix (Thermo Fisher Scientific, Inc.). The RCR thermocycling conditions were as follows: $50^{\circ} \mathrm{C}$ for $3 \mathrm{~min}, 40$ cycles of $\left(95^{\circ} \mathrm{C}\right.$ for $3 \mathrm{~min}, 95^{\circ} \mathrm{C}$ for $10 \mathrm{~s}$, and $60^{\circ} \mathrm{C}$ for $30 \mathrm{~s}$ ), followed by $72^{\circ} \mathrm{C}$ for $5 \mathrm{~min}$. GAPDH was used as the reference gene. Primer sequences are listed in Table I. Relative gene expression was calculated using the $2^{-\Delta \Delta \mathrm{Cq}}$ method (26). All experiments were repeated three times.

Statistical analysis. All results are presented as the mean \pm standard error. Differences between groups were evaluated by Student's t-test. Statistical analysis of differences between groups was performed using SPSS software (version 22; IBM Corp., Armonk, NY, USA) and P<0.05 was considered to indicate a statistically significant difference. Graphs were obtained using Prism (version 5; GraphPad Software, Inc., La Jolla, CA, USA).

\section{Results}

Identification of DEGs and hierarchical cluster analysis. Using the cutoff criteria of adj.p.val $<0.01$ and $\log _{2} \mathrm{FCl}>2,928$ DEGs were identified in human NB cell lines compared with RPE cell lines. Among the 928 DEGs identified, 386 DEGs were upregulated and 542 DEGs were downregulated. The number of downregulated DEGs was 1.4-fold increase compared with upregulated DEGs. Additionally, hierarchy cluster analysis suggested that the DEGs clustered according to the two types of cell lines based on their expression profiles, suggesting that the DEGs may be used for further analyses (Fig. 1).

Functional enrichment analysis of DEGs. DEGs were analyzed using DAVID software to identify significant KEGG pathways and GO terms in the category of BP, CC and MF. The top five GO terms within BP, CC and MF, and the top five KEGG pathways for upregulated and downregulated DEGs are presented in Table II and in Fig. 2. Upregulated genes were significantly enriched in 'neuron migration' [GO term: GO:0001764; including GATA binding protein 2 (GATA2), paired like homeobox $2 \mathrm{~B}$ (PHOX2B) and tubulin $\beta 2 \mathrm{~B}$ class $\mathrm{IIb}$ (TUBB2B)] and 'dopaminergic synapse' signaling pathways [KEGG pathway: hsa04728; including mitogen-activated protein kinase 10 (MAPK10), dopa decarboxylase (DDC) and G protein subunit $\gamma 2$ (GNG2)]. Although 'nervous system development' was more significant, there were more hub genes in PPI network enriched in 'neuron migration' and the P-values
Table I. Primer sequences for the validated genes.

\begin{tabular}{ll}
\hline Primer name & \multicolumn{1}{c}{ Primer sequence (5'-3') } \\
\hline ITGA2-F & GTGGCTTTCCTGAGAACCGA \\
ITGA2-R & GATTCCCACATTGCTGTGCC \\
MAPK10-F & TGGTGACACGTTATTACAGAGC \\
MAPK10-R & GGCCGATTCTCCACATAGTTTCT \\
TUBB2B-F & TACTTTAGGTGTGCGCTGGG \\
TUBB2B-R & GAGGACACCATTCCGACACA \\
RALS11B-F & CGGTTCCTCACCAAACGATTC \\
RALS11B-R & GGCTGTTCTCATGGACCTGAA \\
GAPDH-F & TGACAACTTTGGTATCGTGGAAGG \\
GAPDH-R & AGGCAGGGATGATGTTCTGGAGAG
\end{tabular}

MAPK10, mitogen-activated protein kinase 10; TUBB2B, tubulin $\beta$ 2B class IIb; RASL11B, RAS like family 11 member B; ITGA2, integrin subunit $\alpha 2 ; \mathrm{F}$, forward; R, reverse.

of these two terms were very close. Therefore, 'neuron migration' was selected for further discussion. Besides, we choose 'dopaminergic synapse' pathway to discuss due to this pathway theoretically may be more associated with the development of NB and can be explained by several appropriate article than 'alcoholism' pathway and its significant P-value was only next to 'alcoholism'. The majority of the downregulated DEGs were primarily involved in functions associated with 'focal adhesion' [GO term: GO:0005925; including integrin subunit $\alpha 2$ (ITGA2), ITGA3, ITGA4 and ITGA5] and 'proteoglycans in cancer' (KEGG pathway: hsa05205; ITGA2, ITGB5, CD44 molecule, ITGA5 and thrombospondin 1). Based on the results, 'cell surface' was more significant and its meaning was broader than that of 'focal adhesion'. What' more, the majority of nodes in the following module network belonged to the integrin $\alpha$ and $\beta$ chain family, which were also enriched in this pathway.

PPI network analysis. By analyzing the upregulated DEGs with the STRING database, a PPI network of upregulated DEGs containing 210 nodes and 346 edges was obtained (Fig. 3), whereas, 374 nodes and 1,389 edges were involved in the PPI network of downregulated DEGs (Fig. 4). Based on the connectivity degree among the nodes, the top 20 nodes in the upregulated and downregulated PPI networks are listed in Table III, including MAPK10, TUBB2B, RAS like family 11 member B (RASL11B) and ITGA2.

Module analysis. Using a cutoff of score $>10$, the module analysis demonstrated that only one submodule (submodule 1) was obtained from the downregulated PPI network, whereas no submodule from the upregulated PPI network was obtained. The submodule 1 contained 13 nodes and 77 interactions (Fig. 5). Notably, the majority of nodes in this module belong to the integrin $\alpha$ and $\beta$ chain family.

TF prediction and regulatory network analysis. The DEGs were analyzed using the TRRUST database. Subsequently, 23 DEGs were identified to be TFs. A TF-target regulatory 
Table II. Top five enriched MF, CC and BP GO terms, and KEGG pathways for upregulated DEGs and downregulated DEGs.

A, Upregulated DEGs

\begin{tabular}{|c|c|c|c|c|}
\hline Category & Term & Count & P-value & Examples of genes \\
\hline KEGG & hsa05034:Alcoholism & 11 & $2.84 \times 10^{-4}$ & $\begin{array}{l}\text { HIST1H3J, HIST1H3F, HIST2H4A, GNG4 and } \\
\text { HIST1H3I }\end{array}$ \\
\hline KEGG & hsa04728:Dopaminergic synapse & 9 & $6.07 \times 10^{-4}$ & DDC, KIF5A, GNG2, MAPK10 and GNG4 \\
\hline KEGG & $\begin{array}{l}\text { hsa00330:Arginine and proline } \\
\text { metabolism }\end{array}$ & 6 & $8.31 \times 10^{-4}$ & CKMT1A, CKMT1B, ARG2, MAOA and ALDH2 \\
\hline KEGG & hsa04725:Cholinergic synapse & 8 & $1.27 \times 10^{-3}$ & GNAO1, CHRNB4, SLC18A3, GNG2 and CHRNA7 \\
\hline KEGG & hsa05032:Morphine addiction & 7 & $2.27 \times 10^{-3}$ & GNAO1, PDE3B, PDE10A, GNG2 and GNG4 \\
\hline MF & $\begin{array}{l}\text { GO:0015276:Ligand-gated ion } \\
\text { channel activity }\end{array}$ & 7 & $4.32 \times 10^{-5}$ & $\begin{array}{l}\text { CHRNB4, CHRNA7, CHRNB2, CHRFAM7A } \\
\text { and CHRNA3 }\end{array}$ \\
\hline $\mathrm{MF}$ & GO:0042166:Acetylcholine binding & 6 & $9.4 \times 10^{-5}$ & $\begin{array}{l}\text { CHRNB4, SLC18A3, CHRNA7, CHRNB2 and } \\
\text { CHRNA3 }\end{array}$ \\
\hline MF & $\begin{array}{l}\text { GO:0004889:Acetylcholine-activated } \\
\text { cation-selective channel activity }\end{array}$ & 5 & $7.32 \times 10^{-4}$ & $\begin{array}{l}\text { CHRNB4, CHRNA7, CHRNB2, CHRFAM7A and } \\
\text { CHRNA3 }\end{array}$ \\
\hline MF & $\begin{array}{l}\text { GO:0015464:Acetylcholine receptor } \\
\text { activity }\end{array}$ & 5 & $7.32 \times 10^{-4}$ & $\begin{array}{l}\text { CHRNB4, CHRNA7, CHRNB2, CHRFAM7A and } \\
\text { CHRNA3 }\end{array}$ \\
\hline MF & GO:0008017:Microtubule binding & 12 & $1.57 \times 10^{-3}$ & KIF1A, KIF5B, KIF5A, MAPT and KIF5C \\
\hline $\mathrm{CC}$ & GO:0030424:Axon & 23 & $1.18 \times 10^{-10}$ & DDC, RET, SYT4, STMN2 and ATL1 \\
\hline $\mathrm{CC}$ & GO:0043005:Neuron projection & 23 & $4.20 \times 10^{-10}$ & CADM1, RAB39B, STMN2, KIF5A and SLC6A2 \\
\hline $\mathrm{CC}$ & GO:0030054:Cell junction & 30 & $6.16 \times 10^{-9}$ & SEPT3, SYT4, GABRB3, GRIK2 and SYP \\
\hline $\mathrm{CC}$ & GO:0030426:Growth cone & 11 & $5.07 \times 10^{-5}$ & TSHZ3, STMN2, MAPT, LRRTM1 and STMN4 \\
\hline $\mathrm{CC}$ & GO:0044295:Axonal growth cone & 5 & $2.67 \times 10^{-4}$ & FLRT3, KIF5B, PTCH1, L1CAM and OLFM1 \\
\hline BP & $\begin{array}{l}\text { GO:0007399:Nervous system } \\
\text { development }\end{array}$ & 21 & $6.05 \times 10^{-7}$ & SCN3B, FGF14, DPYSL5, L1CAM and CNTFR \\
\hline $\mathrm{BP}$ & GO:0001764:Neuron migration & 13 & $7.00 \times 10^{-7}$ & ASCL1, GATA2, PHOX2B, TUBB2B and GATA3 \\
\hline $\mathrm{BP}$ & $\begin{array}{l}\text { GO:0035095:behavioral response } \\
\text { to nicotine }\end{array}$ & 5 & $1.48 \times 10^{-5}$ & $\begin{array}{l}\text { CHRNB4, CHRNA7, CHRNB2, CHRFAM7A } \\
\text { and CHRNA3 }\end{array}$ \\
\hline BP & GO:0060384:Innervation & 6 & $1.65 \times 10^{-5}$ & RET, SULF2, GABRB3, RNF165 and ISL1 \\
\hline BP & $\begin{array}{l}\text { GO:0048485:Sympathetic nervous } \\
\text { system development }\end{array}$ & 5 & $1.46 \times 10^{-4}$ & PHOX2A, PHOX2B, ASCL1, HAND2 and GATA3 \\
\hline
\end{tabular}

B, Downregulated DEGs

\begin{tabular}{lllll}
\hline Category & \multicolumn{1}{c}{ Term } & Count & P-value & \multicolumn{1}{c}{ Examples of genes } \\
\hline KEGG & hsa05205:Proteoglycans in cancer & 27 & $8.81 \times 10^{-10}$ & ITGA2, ITGB5, CD44, ITGA5 and THBS1 \\
KEGG & hsa04512:ECM-receptor interaction & 17 & $1.25 \times 10^{-8}$ & COL4A2, COL4A1, ITGA11, ITGA2 and ITGB5 \\
KEGG & hsa04510:Focal adhesion & 25 & $3.67 \times 10^{-8}$ & ITGA11, ITGA2, ITGB5, ITGA3 and ITGA4 \\
KEGG & hsa05412:Arrhythmogenic right & 14 & $3.21 \times 10^{-7}$ & ITGA11, ITGA2, GJA1, ITGB5 and ITGA3 \\
& ventricular cardiomyopathy (ARVC) & & & \\
KEGG & hsa05410:Hypertrophic cardiomyopathy & 14 & $9.98 \times 10^{-7}$ & IL6, ITGB8, ITGA5, ITGAV and ITGA7 \\
& (HCM) & & & \\
MF & GO:0001968:Fibronectin binding & 11 & $6.48 \times 10^{-10}$ & CTSK, CTGF, ITGAV, CCDC80 and FSTL3 \\
MF & GO:0005518:Collagen binding & 14 & $5.31 \times 10^{-9}$ & ADGRG6, ITGA11, ITGA2, SMAD3 and ITGA3 \\
MF & GO:0005509:Calcium ion binding & 49 & $5.65 \times 10^{-9}$ & LTBP1, LTBP2, LTBP3, FSTL1 and EDIL3 \\
MF & GO:0005178:Integrin binding & 17 & $2.01 \times 10^{-8}$ & FBN1, ITGA2, ITGA3, EDIL3 and CD151 \\
MF & GO:0008201:Heparin binding & 19 & $3.16 \times 10^{-7}$ & BMP4, CCL2, LTBP2, LXN and FBN1 \\
CC & GO:0009986:Cell surface & 59 & $1.21 \times 10^{-19}$ & MICB, MICA, CSPG4, TLR3 and TLR4 \\
CC & GO:0005925:Focal adhesion & 50 & $1.40 \times 10^{-19}$ & ITGA11, ITGA2, ITGB5, ITGA3 and ITGA4 \\
CC & GO:0031012:Extracellular matrix & 41 & $2.48 \times 10^{-17}$ & LTBP1, LTBP2, LTBP3, IGFBP7 and POSTNS \\
CC & GO:0005886:Plasma membrane & 184 & $4.83 \times 10^{-14}$ & F2RL2, SLC9A7, MICB, MICA and VCL \\
CC & GO:0070062:Extracellular exosome & 135 & $4.58 \times 10^{-12}$ & CHMP4C, LTBP2, LTBP3, CSPG4 and FSTL1
\end{tabular}


Table II. Continued.

\begin{tabular}{llccl}
\multicolumn{2}{l}{ B, Downregulated DEGs } \\
\hline \multicolumn{1}{c}{ Category } & \multicolumn{1}{c}{ Term } & Count & P-value & \multicolumn{1}{c}{ Examples of genes } \\
\hline BP & $\begin{array}{l}\text { GO:0007155:Cell adhesion } \\
\text { BP }\end{array}$ & 51 & $6.51 \times 10^{-17}$ & IGFBP7, POSTN, EDIL3, CD151 and VCL \\
GP & $\begin{array}{l}\text { organization } \\
\text { GO:0035987:Endodermal cell }\end{array}$ & 13 & $2.05 \times 10^{-12}$ & HMGA2, COL8A1, MMP14, MMP2 and FN1 \\
BP & $\begin{array}{l}\text { differentiation } \\
\text { GO:0010628:Positive regulation } \\
\text { of gene expression }\end{array}$ & 32 & $8.90 \times 10^{-12}$ & ACTA2, SMAD3, ITGA3, INHBA and TFAP2A \\
BP & $\begin{array}{l}\text { GO:0001666:Response to hypoxia } \\
\text { ITGA1, ITGB5, POSTN, ABI3BP and CD44 }\end{array}$ & 23 & $2.27 \times 10^{-9}$ & MMP14, MMP2, TGFB1, TGFB2 and THBS1
\end{tabular}

DEGs, differentially expressed genes; MF, molecular function; CC, cellular component; BP, biological process; KEGG, Kyoto Encyclopedia of Genes and Genomes; GO, Gene Ontology.

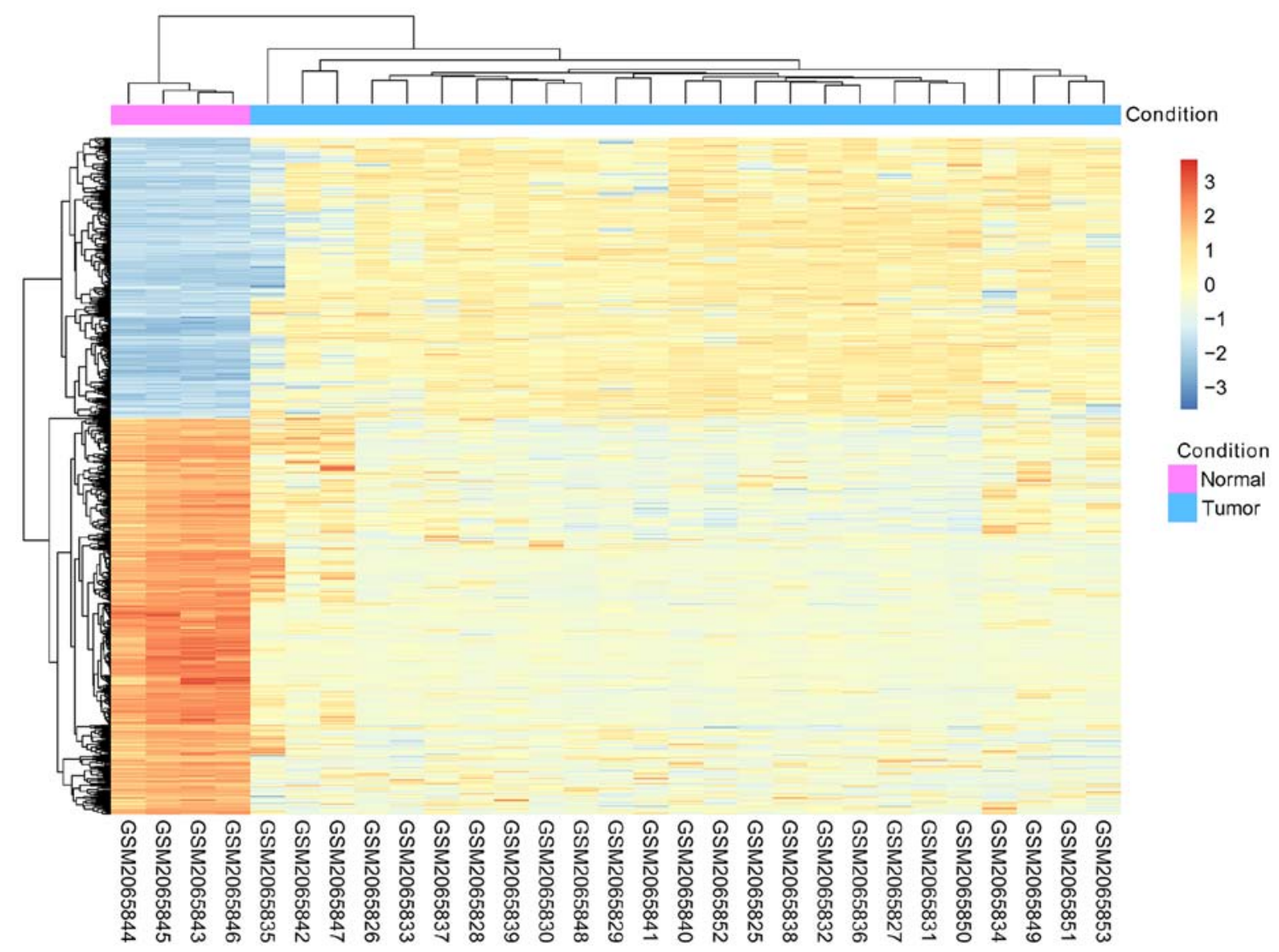

Figure 1. Hierarchical cluster map of DEGs. Horizontal axis indicates the names of each sample, whereas, the right vertical axis indicates clusters of DEGs. Gradual change from blue to orange indicates the expression values, from decreased to increased expression levels. DEGs, differentially expressed genes.

network consisting of 73 nodes (23 TFs and 50 DEGs) and 52 interaction associations, such as PHOX2B-ALK was constructed (Fig. 6). The top 20 nodes with high degrees are presented in Table IV and include transcription factor AP-2 $\alpha$, melanocyte inducing transcription factor, SMAD family member 3 (SMAD3), runt related transcription factor 1 and PHOX2B. Notably, SMAD3 and PHOX2B belong to the top 20 DEGs in the PPI network. Additionally, the number of downregulated TFs was higher compared with upregulated TFs.

Validation of gene expression. RT-qPCR was conducted to test the expressions of DEGs. A total of four DEGs were validated: ITGA2, MAPK10, TUBB2B and RASL11B, which have not previously been associated with NB. Compared with hTERT-RPE cells, MAPK10, TUBB2B and RASL11B 
A

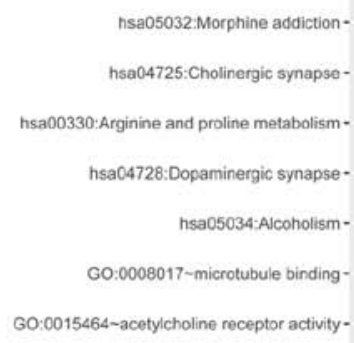

GO:0004889 acetylcholne-activated cation-selective channel activity-

G0:0042166 acetylcholine binding -

$\stackrel{E}{\varrho}$

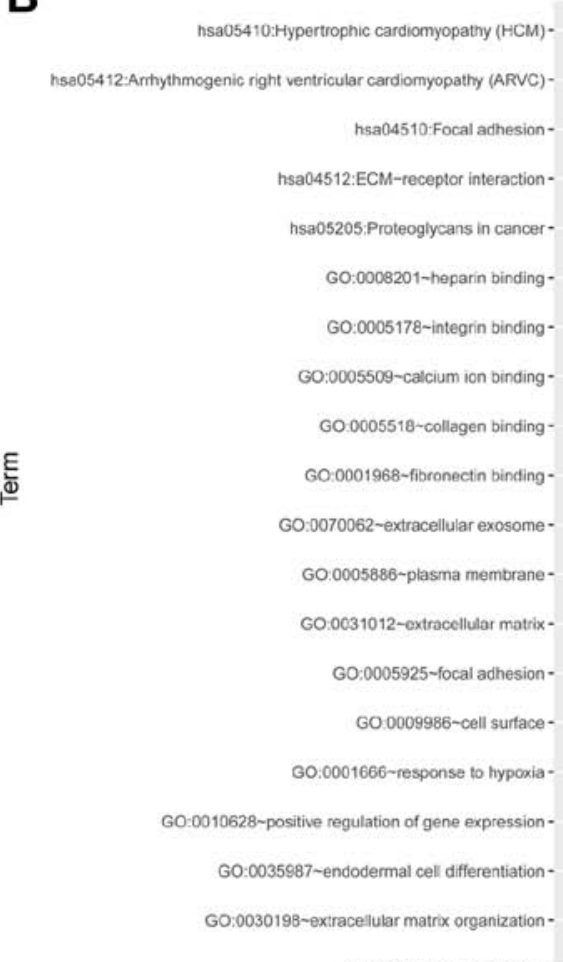

G0:0007155-cell achesion -

GO:0030426-growth cone-

GO:0030054-cell junction -

G0:0043005-neuron projection-

GO:0030424 axon -

GO:0048485 sympathetic nervous system development-

GO0060384-innervation-

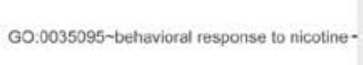

GO:0001764 neuron migration -

G0:0007399-nervous system development-
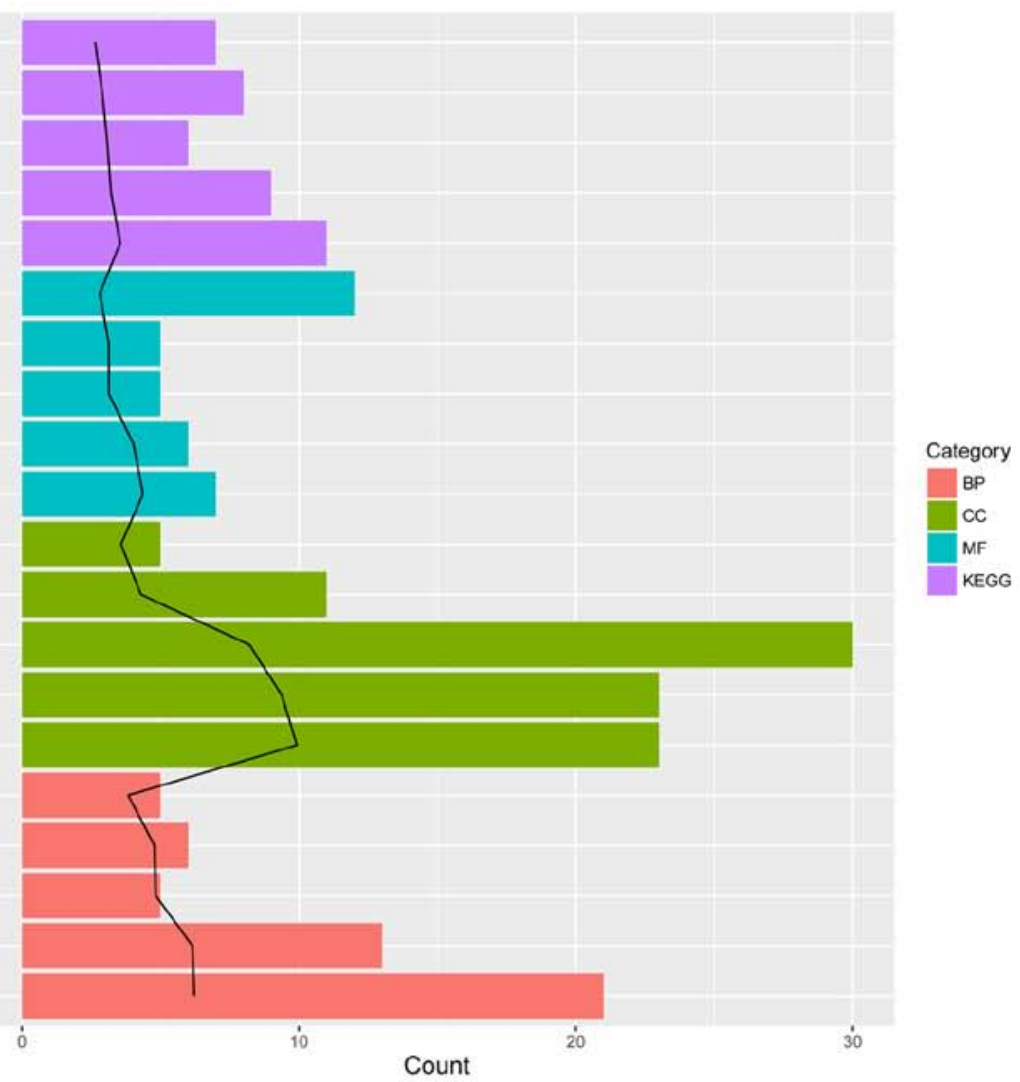

Count

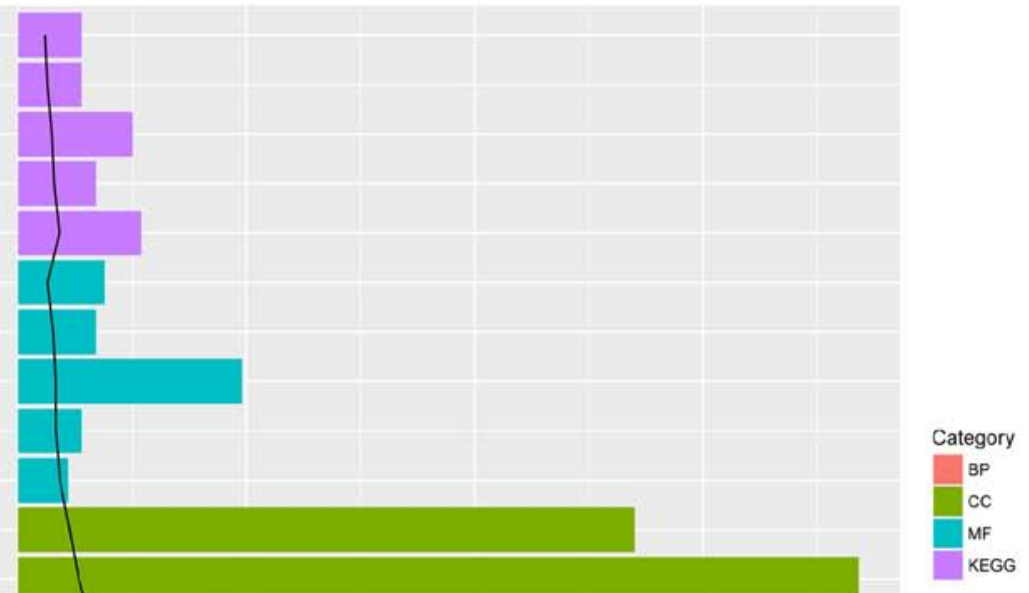

KEGG

Figure 2. Top five enriched groups in each functional enrichment analysis. (A) Upregulated DEGs. (B) Downregulated DEGs. MF, molecular function; CC, cellular component; BP, biological process; KEGG, Kyoto Encyclopedia of Genes and Genomes; DEGs, differentially expressed genes; GO, Gene Ontology.

exhibited a significantly increased expression level in SH-SY5Y cells (Fig. 7A-C). In contrast, the mRNA expression level of ITGA2 was decreased in human SH-SY5Y cells
(Fig. 7D) compared with hTERT-RPE cells. Notably, the RT-qPCR results confirmed the bioinformatics analysis of DEGs in the GSE78061 dataset. 


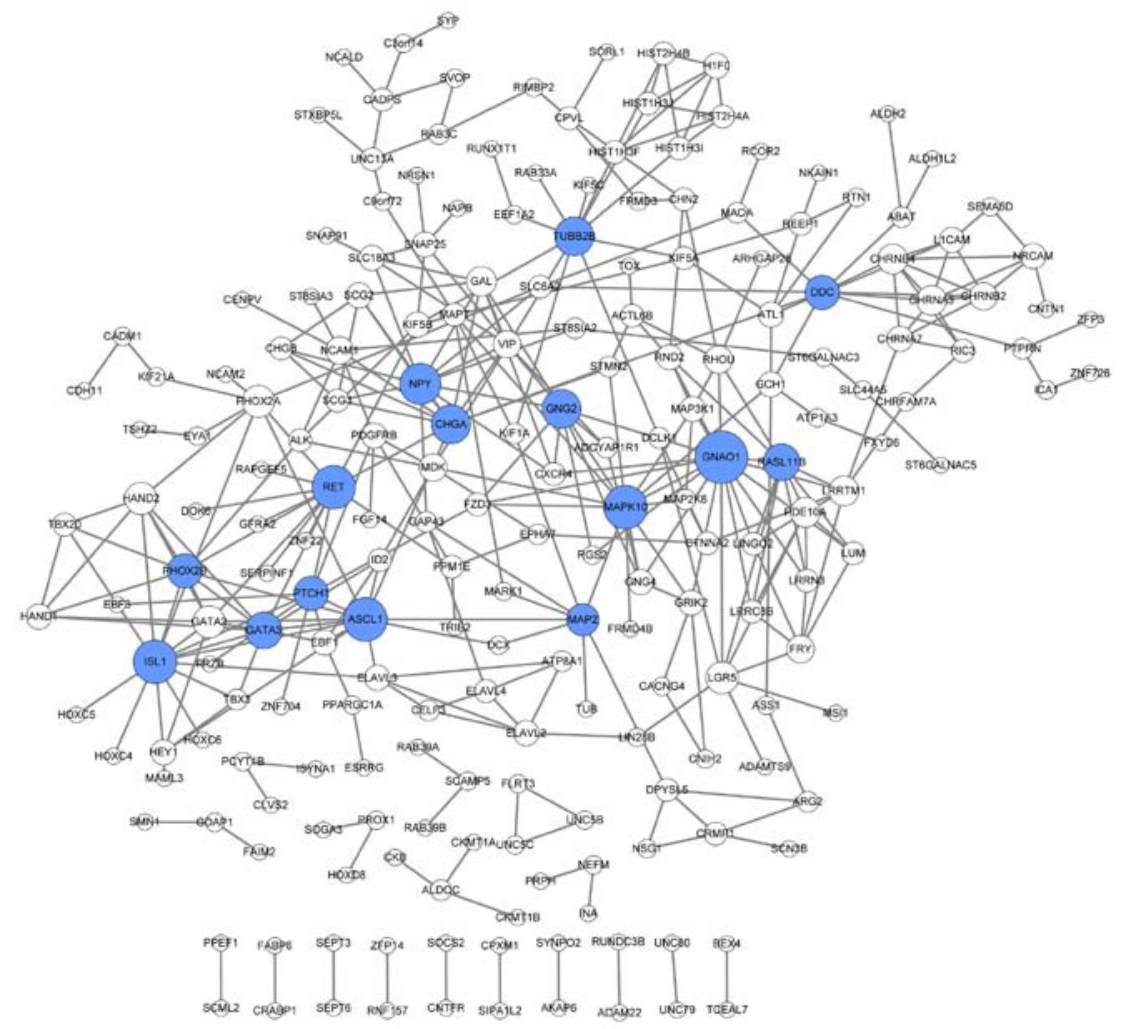

Figure 3. Protein-protein interaction network of upregulated differentially expressed genes. The top 15 nodes ranked by connectivity degrees are highlighted in blue. Nodes represent genes, and edges connect the nodes to indicate their associations. The node size represents connectivity degree.

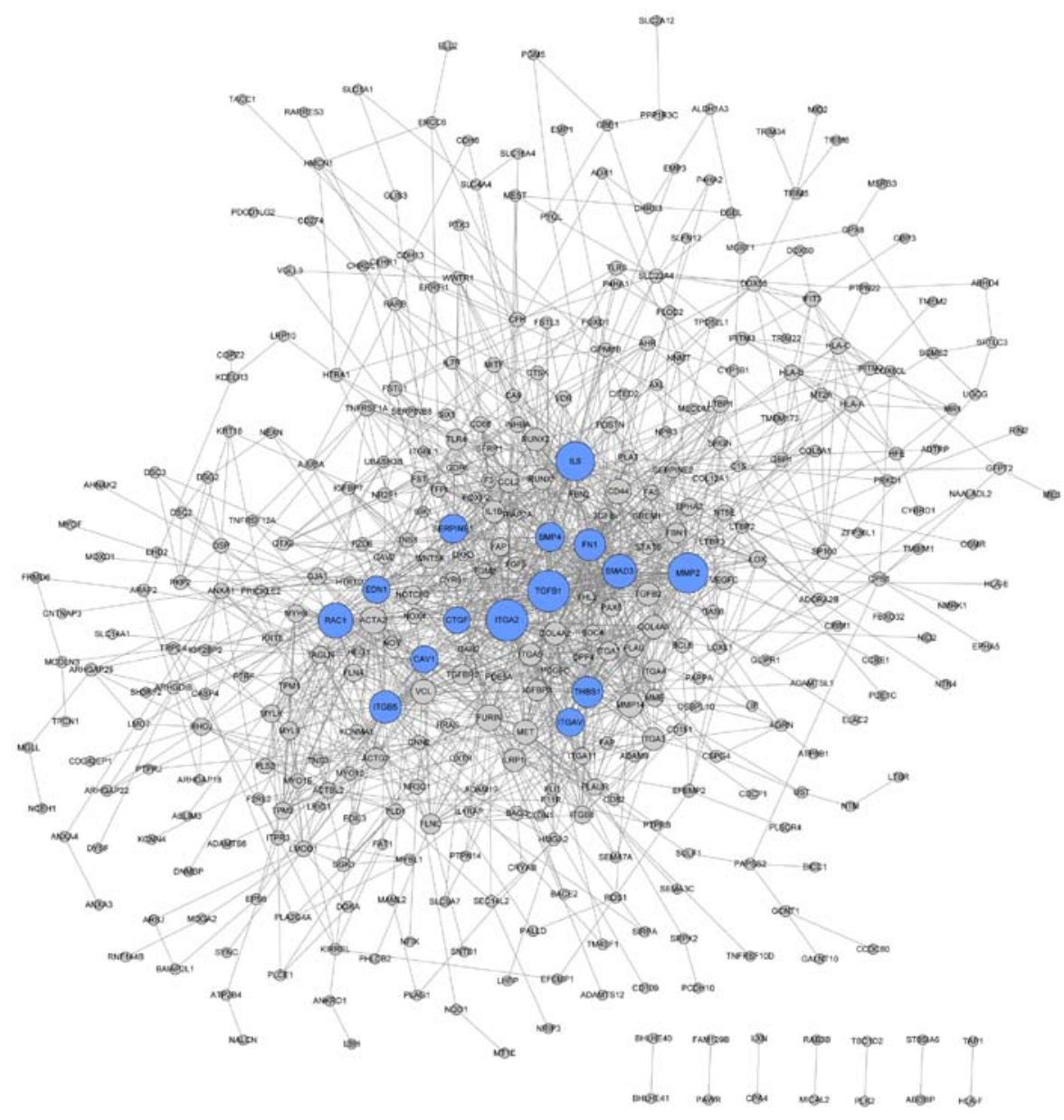

Figure 4. Protein-protein interaction network for downregulated differentially expressed genes. The top 15 nodes ranked by degree values are highlighted in blue. Nodes represent genes, and edges connecting the nodes indicate their associations. The node size represents the respective connectivity degree. 
Table III. Topological property scores for nodes in the protein-protein interaction network, top 20 upregulated DEGs and top 20 downregulated DEGs.

\begin{tabular}{|c|c|}
\hline Node & Degree \\
\hline GNAO1 & 17 \\
\hline ISL1 & 13 \\
\hline MAPK10 & 13 \\
\hline ASCL1 & 13 \\
\hline RET & 13 \\
\hline NPY & 12 \\
\hline TUBB2B & 11 \\
\hline GNG2 & 11 \\
\hline CHGA & 11 \\
\hline RASL11B & 10 \\
\hline GATA3 & 10 \\
\hline PTCH1 & 9 \\
\hline PHOX2B & 9 \\
\hline DDC & 9 \\
\hline MAP2 & 8 \\
\hline LGR5 & 8 \\
\hline GATA2 & 8 \\
\hline PHOX2A & 8 \\
\hline HAND2 & 8 \\
\hline PDE10A & 7 \\
\hline
\end{tabular}

B, Downregulated DEGs

\begin{tabular}{lc}
\hline Node & Degree \\
\hline ITGA2 & 58 \\
TGFB1 & 58 \\
MMP2 & 55 \\
IL6 & 53 \\
RAC1 & 46 \\
SMAD3 & 44 \\
ITGB5 & 41 \\
FN1 & 40 \\
THBS1 & 39 \\
BMP4 & 35 \\
SERPINE1 & 35 \\
ITGAV & 34 \\
EDN1 & 32 \\
CAV1 & 32 \\
CTGF & 31 \\
ACTA2 & 31 \\
FURIN & 30 \\
CD44 & 28 \\
MMP14 & 27 \\
MET & 27 \\
\hline
\end{tabular}

DEGs, differentially expressed genes.
Table IV. Topological property scores for the top 20 nodes in the TF-target regulatory network.

A, Upregulated DEGs

\begin{tabular}{lcc}
\hline Node & Description & Degree \\
\hline HEY1 & TF & 4 \\
PHOX2B & TF & 2 \\
HOXC6 & TF & 2 \\
\hline
\end{tabular}

B, Downregulated DEGs

\begin{tabular}{llc}
\hline Node & Description & Degree \\
\hline TFAP2A & TF & 9 \\
MITF & TF & 8 \\
SMAD3 & TF & 6 \\
RUNX1 & TF & 5 \\
PAX6 & TF & 5 \\
AHR & TF & 5 \\
VDR & TF & 4 \\
RUNX2 & TF & 4 \\
FOXP2 & TF & 4 \\
FLI1 & TF & 4 \\
MMP2 & Target & 3 \\
MET & Target & 3 \\
IGFBP3 & Target & 3 \\
IL6 & Target & 3 \\
WWTR1 & TF & 2 \\
OTX2 & TF & 2 \\
NR3C1 & TF & 2 \\
\hline
\end{tabular}

TF, transcription factor; DEGs, differentially expressed genes.

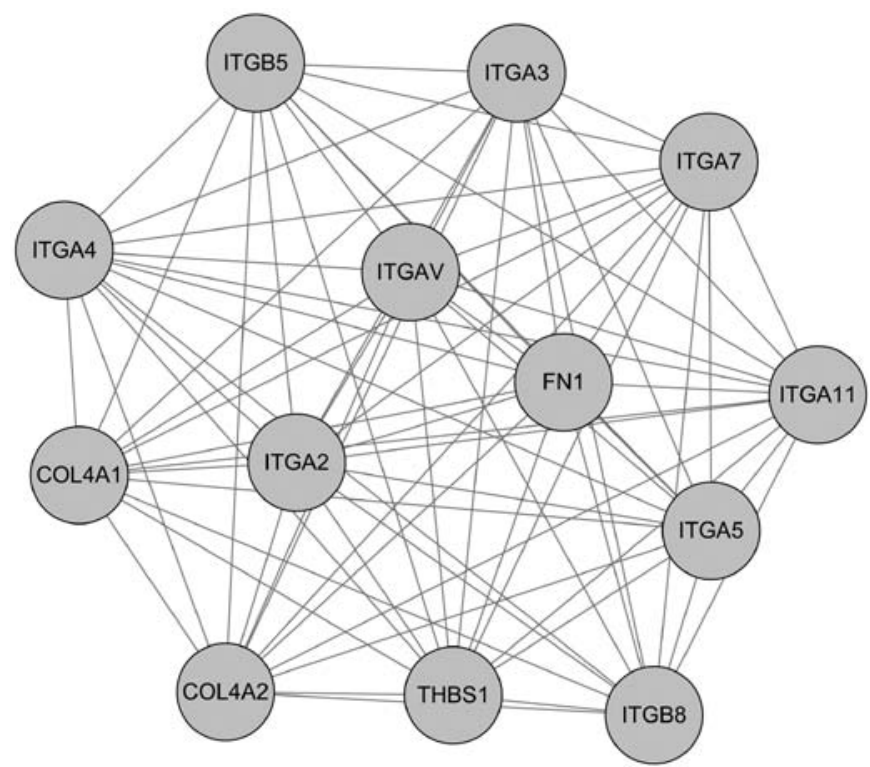

Figure 5. Result for module analysis. ITGA, integrin subunit $\alpha$; ITGB, integrin subunit $\beta$; COL4A1, collagen type IV $\alpha 1$ chain; FN1, fibronectin 1; THBS1, thrombospondin 1. 


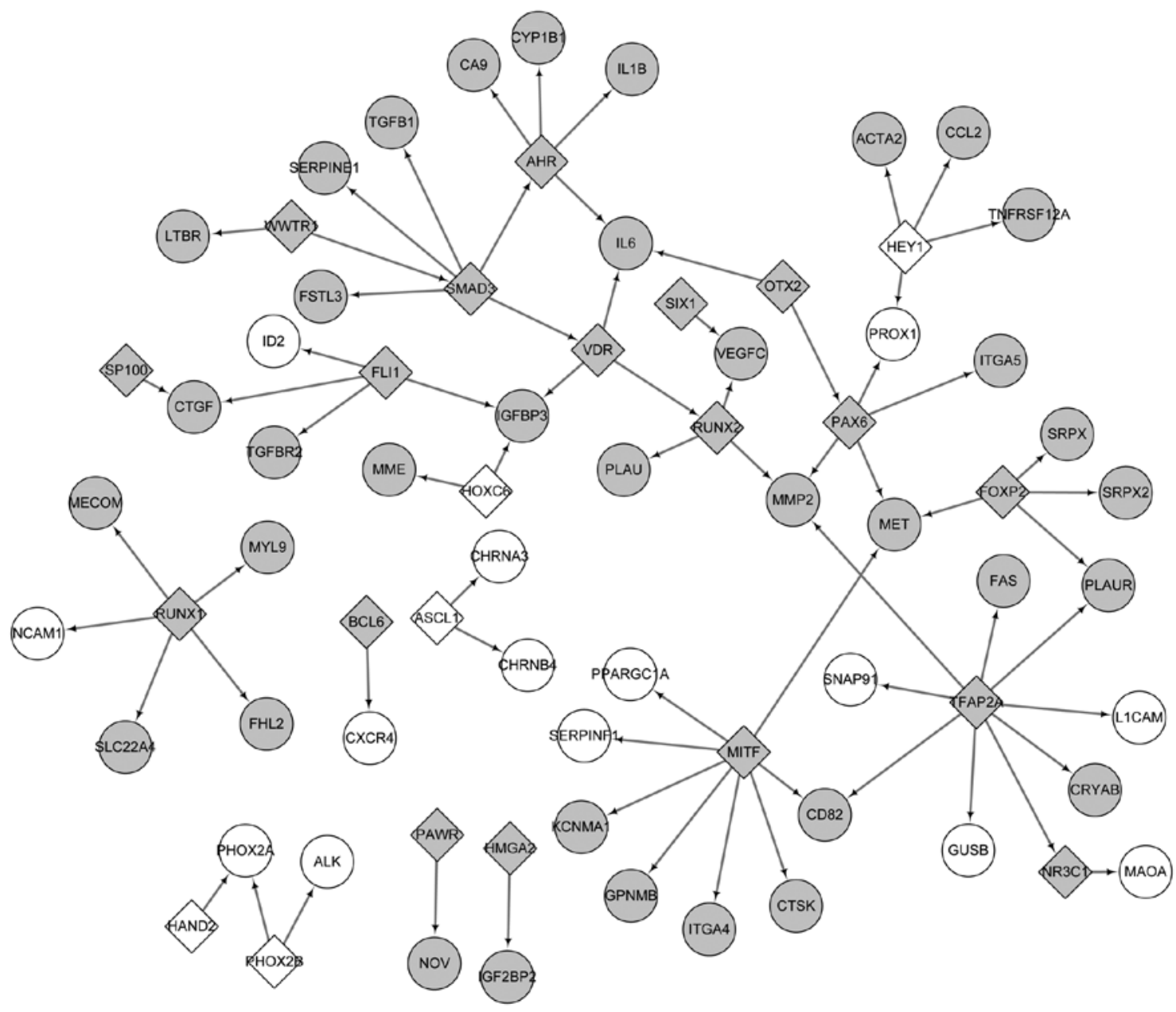

Figure 6. Integrative regulatory network of TF-targets. The diamond-shaped nodes represent TFs and circles represent target genes. Gray indicates downregulated genes and white indicates upregulated genes. TF, transcription factor.
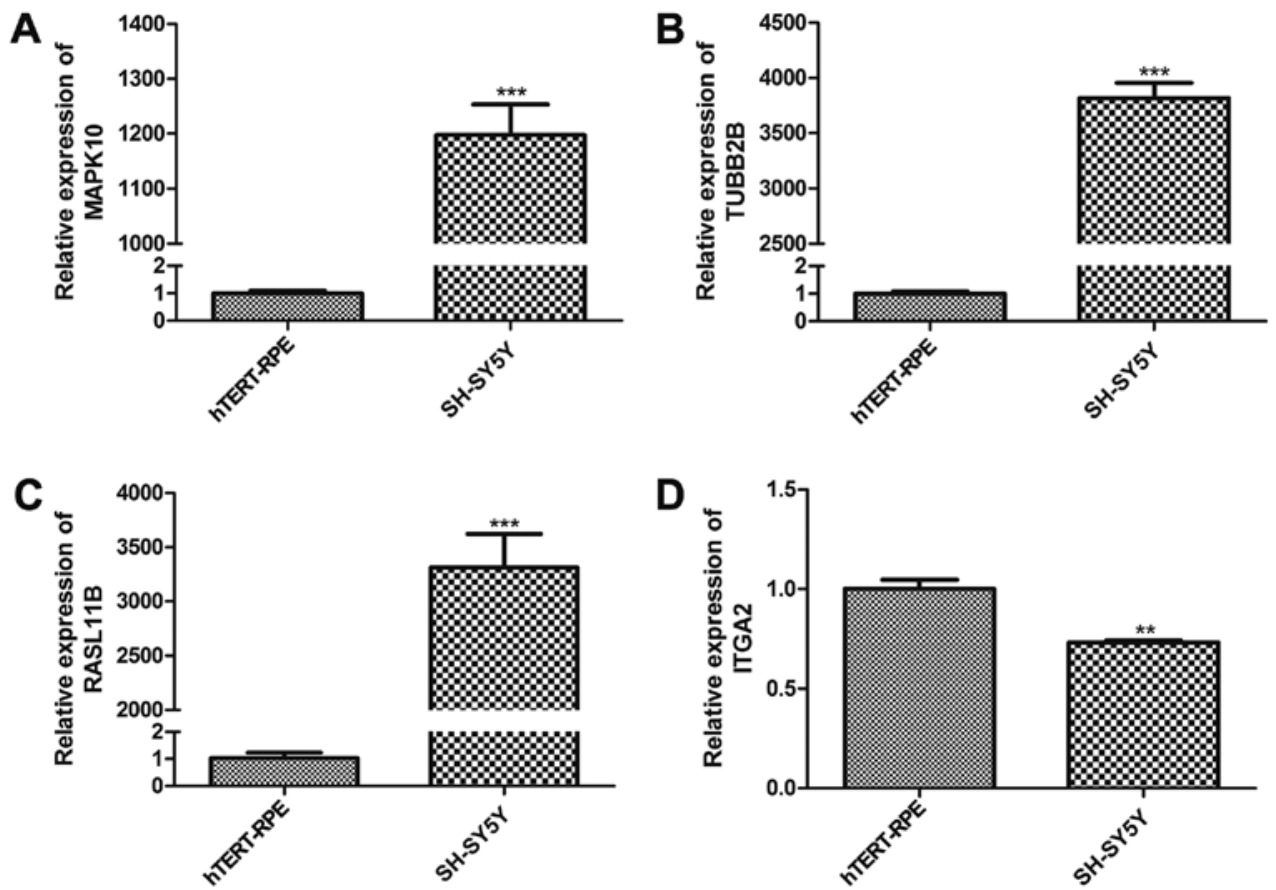

Figure 7. RT-qPCR analysis. Relative mRNA expressions of (A) MAPK10, (B) TUBB2B, (C) RASL11B and (D) ITGA2 in the human neuroblastoma SH-SY5Y cell line compared with the hTERT-RPE cell line detected by RT-qPCR. ${ }^{* *} \mathrm{P}<0.01,{ }^{* * * *} \mathrm{P}<0.001$ vs. hTERT-RPE cells. RT-qPCR, reverse transcription-quantitative polymerase chain reaction; MAPK10, mitogen-activated protein kinase 10; TUBB2B, tubulin $\beta$ 2B class IIb; RASL11B, RAS like family 11 member B; ITGA2, integrin subunit $\alpha 2$. 


\section{Discussion}

In the present study, a set of 928 DEGs was identified in NB cell lines compared with RPE cells lines, 386 DEGs were upregulated and 542 DEGs were downregulated. Upregulated DEGs were significantly associated with 'neuron migration' (including PHOX2B and TUBB2B) and 'dopaminergic synapse' signaling pathways (including MAPK10, DDC and GNG2). The majority of downregulated DEGs were primarily involved in "focal adhesion' (including ITGA2 and ITGA3). Additionally, the DEGs MAPK10, DDC, GNG2, PHOX2B, TUBB2B, RASL11B, and ITGA2 were highlighted in the PPI networks. Furthermore, based on regulatory associations reported in the TRRUST database, PHOX2B was predicted to function as a TF upstream of ALK in the regulatory network. The upregulation of MAPK10, TUBB2B and RASL11B, and the downregulation of ITGA2 were detected by RT-qPCR in NB SH-SY5Y cells, consistent with the present bioinformatics analysis.

In the present study, a number of hub genes, including MAPK10, DDC and GNG2, belonging to the upregulated PPI networks, were predicted to be involved in the 'dopaminergic synapse' signaling pathway. The majority of human NB cell lines, including the SK-N-AS cell line, are dopaminergic NB cells and exhibit characteristics of dopaminergic neurons (27). Additionally, $\sim 80 \%$ of patients diagnosed with NB exhibit increased expression levels of catecholamine and its metabolites, including dopamine and epinephrine, making these molecules promising tumor markers for diagnosing NB (28). Therefore, alterations in the dopamine expression levels mediated by its associated signaling pathway may serve an important role in NB development.

Similarly, $D D C$ encodes for an enzyme associated with dopamine synthesis and its expression was demonstrated to be significantly increased in patients with NB (29). Phospholipase $\mathrm{C} \eta 2$ (PLCH2) belongs to the PLC- $\eta$ family, and is part of a crucial intracellular signaling system associated with neurotransmitter signal transduction, synapse formation and neuronal network formation; however it is additionally involved in the development of NB and other central nervous system-associated tumors (30). Notably, a previous study suggested that PLCH2 was activated by GNG2 (31), and GNG2 was expressed in the fetal tissues, adrenal gland and brain, regulating a series of intracellular effectors, including ion channels and adenylyl cyclase (32), which were previously associated with NB $(33,34)$. Therefore, the present results, suggesting that DDC and GNG2 were associated with NB, are consistent with the results of previous studies.

Additionally, MAPK is a member of the serine/threonine protein kinase family and serves crucial roles in regulating neuronal processes at the cellular level, including synaptic transmission, morphological differentiation and survival (35). Furthermore, Morón et al (36) suggested that MAPK regulated dopamine transporters, modulating the concentration of dopamine in the extracellular space. Furthermore, a previous study demonstrated that MAPK10 was associated with the development of cancer (37). Notably, MAPK10 and MAPK9 serve important roles in the death of dopamine neurons of the substantia nigra (38). Similarly, a previous study demonstrated that activation of the MAPK/Jun proto-oncogene (Jun) signaling pathway induced apoptosis in SK-N-SH NB cells by regulating the targets of the adaptor related protein complex 1 complex (39). MAPK10, as a MAPK family member, is additionally involved in the MAPK/Jun signaling pathway. Therefore, MAPK10 may be associated with the progression of NB by serving a role in the dopaminergic synapse and MAPK/Jun signaling pathways. As expected, the expression level of MAPK10 was significantly higher in human NB SH-SY5Y cell lines compared with hTERT-RPE cell lines.

In the present study, it was identified that TUBB2B and RASL11B were significantly upregulated in human NB SH-SY5Y cells. TUBB2B, a principal component of microtubules, is synthesized in axons and is involved in neuronal migration (40). TUBB2B served an essential role in the neural development system and served as a primary neuron marker (41). TUBB2B was identified to be upregulated in the brain cortex tissues of a microtubule associated protein $\tau$ (MAPT) transgenic mouse model of Alzheimer's disease, suggesting an interaction between MAPT and TUBB2B (42). Notably, hyperphosphorylated MAPT during mitosis is associated with aberrant cell-cycle-dependent kinase activity in the NB cell line SH-SY5Y (43). Therefore, in the present study it was hypothesized that the dysregulation of TUBB2B may be responsible for aberrant neuronal migration and abnormal cell cycle during the development of NB. RASL11B belongs to the small GTPase family and Ras subfamily, and serves as a tumor suppressor gene (44). Additionally, RASL11B was demonstrated to be associated with inflammation and cancer through involvement of transforming growth factor- $\beta 1$-mediated processes (45). Therefore, RASL11B may be associated with the development of NB. However, further studies are required to investigate its molecular mechanism in NB.

In the TF-target regulatory network, a regulatory association between PHOX2B (upregulated DEG) and anaplastic lymphoma receptor tyrosine kinase (ALK; upregulated DEG) was predicted. Upregulation of PHOX2B is associated with MYCN proto-oncogene, bHLH transcription factor (MYCN), and it was demonstrated to contribute to NB cell proliferation (46). Additionally, upregulation of MYCN is a primary cause of NB progression (46). Notably, Bachetti et al (47) identified a correlation between the expression levels of PHOX2B and ALK, by performing small interfering RNA silencing and overexpression experiments. In contrast, ITGA2, which exhibited the highest connectivity degree in the downregulated PPI network, was predicted to be associated with NB. ITGA2 is a member of the cell adhesion receptor family and is crucial for the cell proliferation, metastasis and apoptosis of solid tumors (48). It has been suggested that decreased expression levels of ITAG2 and ITAG3 are inversely correlated with MYCN expression in NB cell lines (49). The present results suggested that ITGA2 and ITGA3 which were present in the GO terms of 'extracellular matrix' and 'focal adhesion' pathways, were significantly downregulated in NB cell lines. Additionally, the present experimental data demonstrated that ITGA2 expression was significantly decreased in SH-SY5Y NB cells. Therefore, PHOX2B, ALK, ITGA2 and ITGA3 may be useful therapeutic targets for NB.

In the present study, although numerous genes were selected, only a limited number of genes (including MAPK10, DDC, GNG2, PHOX2B, TUBB2B and RASL11B) were discussed due to the fact that multiple genes identified in the 
present analysis [including GATA3, Microtubule-associated protein 2 (MAP2), Chromogranin A (CHGA) and NPY] have been previously observed to be associated with the development of NB. GATA3 serves a crucial role in the regulation of cell differentiation and proliferation of SK-N-SH NB cells (50). MAP2 synthesis is increased in NB2a cells, which exhibit dendritic properties in terms of morphology and preferential distribution of MAP2 (51). A previous study demonstrated that CHGA and NPY serve as NB tumor gene markers and are involved in the metastasis of NB cells (52). Notably, these previous findings supported the results of the present study.

However, there were numerous limitations of the present study. Due to the low incidence of NB and the limited possibilities of hospitals in sharing tissues of patients, there were not enough NB tissue samples to be collected in a short-term, in order to perform gene expression validation. Furthermore, NB originates from sympathetic ganglia near the spinal cord and within the adrenal medulla (53). However, hTERT-RPE cells are not part of this tissue, thus hTERT-RPE cells are not the optimal control. This cell line was selected as control cells in the GSE78061 dataset and no appropriate alternative cells were considered. The hTERT-RPE cell line was selected as the control for experiment validation, in order to be consistent with the GSE78061 dataset. Furthermore, due to insufficient funds, the experimental validation was not conducted in additional cell lines. The present findings require confirmation using additional cell lines and the analysis of a sufficient number of NB and normal tissue or adjacent tissue samples.

Collectively, a total of 386 upregulated and 542 downregulated DEGs were identified in NB cell lines. Additionally, MAPK10, DDC, GNG2, PHOX2B, TUBB2B, RASL11B, ITGA2 and ITGA3, which exhibit high connectivity degrees in the PPI networks, may contribute to NB pathogenesis. Furthermore, a novel significant association between the increased expression levels of MAPK10, TUBB2B and RASL11B, and NB was identified.

\section{Acknowledgements}

Not applicable.

\section{Funding}

No funding was received.

\section{Availability of data and materials}

The datasets analyzed during the current study are available in the GEO repository (www.ncbi.nlm.nih.gov/geo/query/acc. cgi?acc=GSE78061).

\section{Authors' contributions}

JTL contributed to the study design, data collection and writing of the manuscript. YLL contributed to the data interpretation and discussion. All authors read and approved the final manuscript.

\section{Ethics approval and consent to participate}

Not applicable.

\section{Patient consent for publication}

Not applicable.

\section{Competing interests}

The authors declare that they have no competing interests.

\section{References}

1. Shohet J and Foster J: Neuroblastoma. BMJ 357: j1863, 2017.

2. Abo-Elenain A, Naiem Y, Hamedhosam-Eldin Hotmail Com $\mathrm{H}$ Emam M, Elkashef W and AbdelRafee A: Right adrenal gland neuroblastoma infiltrating the liver and mimicking mesenchymal hamartoma: A case report. Int J Surg Case Rep 12: 95-98, 2015.

3. Park JR, Eggert A and Caron H: Neuroblastoma: Biology, prognosis, and treatment. Pediatr Clin North Am 55: 97-120, x, 2008.

4. Castleberry RP, Shuster JJ and Smith EI: The Pediatric Oncology Group experience with the international staging system criteria for neuroblastoma. Member Institutions of the Pediatric Oncology Group. J Clin Oncol 12: 2378-2381, 1994.

5. Tonini GP: Growth, progression and chromosome instability of Neuroblastoma: A new scenario of tumorigenesis? BMC Cancer 17: 20, 2017.

6. Galli S, Naranjo A, Van Ryn C, Tilan JU, Trinh E, Yang C, Tsuei J, Hong SH, Wang H, Izycka-Swieszewska E, et al: Neuropeptide $\mathrm{Y}$ as a biomarker and therapeutic target for neuroblastoma. Am J Pathol 186: 3040-3053, 2016.

7. Lee SG, Jeon HY, Su ZZ, Richards JE, Vozhilla N, Sarkar D, Van Maerken T and Fisher PB: Astrocyte elevated gene-1 contributes to the pathogenesis of neuroblastoma. Oncogene 28: 2476-2484, 2009.

8. Brunen D, de Vries RC, Lieftink C, Beijersbergen RL and Bernards R: PIM kinases are a potential prognostic biomarker and therapeutic target in neuroblastoma. Mol Cancer Ther 17: 849-857, 2018

9. Galinski B, Luxemburg M, Ewart M, Landesman Y and Weiser D: Abstract 1938: Exportin-1 (XPO1) is a novel therapeutic biomarker for patients with neuroblastoma. Cancer Res 77: 1938-1938, 2017.

10. Cui X, Yang Y, Jia D, Jing Y, Zhang S, Zheng S, Cui L, Dong R and Dong K: Downregulation of bone morphogenetic protein receptor 2 promotes the development of neuroblastoma. Biochem Biophys Res Commun 483: 609-616, 2017.

11. Ishizuka Y, Koshinaga T, Hirano T, Nagasaki-Maeoka E, Watanabe Y, Hoshi R, Yoshizawa S, Sugito K, Kawashima H, Uekusa S, et al: NRP1 knockdown promotes the migration and invasion of human neuroblastoma-derived SKNAS cells via the activation of $\beta 1$ integrin expression. Int J Oncol 53: 159-166, 2018.

12. Mei Z, Yan P, Wang Y, Liu S and He F: Knockdown of zinc transporter ZIP8 expression inhibits neuroblastoma progression and metastasis in vitro. Mol Med Rep 18: 477-485, 2018.

13. Irshad S, Pedley RB, Anderson J, Latchman DS and Budhram-Mahadeo V: The Brn-3b transcription factor regulates the growth, behavior and invasiveness of human neuroblastoma cells in vitro and in vivo. J Biol Chem 290: 21617-21627, 2015.

14. Souzaki R, Tajiri T, Souzaki M, Kinoshita Y, Tanaka S, Kohashi K, Oda Y, Katano M and Taguchi T: Hedgehog signaling pathway in neuroblastoma differentiation. J Pediatr Surg 45: 2299-2304, 2010.

15. Hart LS, Rader J, Raman P, Batra V, Russell MR, Tsang M, Gagliardi M, Chen L, Martinez D, Li Y, et al: Preclinical therapeutic synergy of MEK1/2 and CDK4/6 inhibition in neuroblastoma. Clin Cancer Res 23: 1785-1796, 2017.

16. Carvalho BS and Irizarry RA: A framework for oligonucleotide microarray preprocessing. Bioinformatics 26: 2363-2367, 2010.

17. Irizarry RA, Hobbs B, Collin F, Beazer-Barclay YD, Antonellis KJ, Scherf U and Speed TP: Exploration, normalization, and summaries of high density oligonucleotide array probe level data. Biostatistics 4: 249-264, 2003.

18. Smyth GK, Ritchie M, Thorne N and Wettenhall J: LIMMA: Linear models for microarray data. In: Bioinformatics and Computational Biology Solutions Using $\mathrm{R}$ and Bioconductor. $\mathrm{SBH}, 2005$.

19. Hulsegge I, Kommadath A and Smits MA: Globaltest and GOEAST: Two different approaches for Gene Ontology analysis. BMC Proc 3 (Suppl 4): S10, 2009. 
20. Kanehisa M and Goto S: KEGG: Kyoto encyclopedia of genes and genomes. Nucleic Acids Res 28: 27-30, 2000.

21. Dennis G Jr, Sherman BT, Hosack DA, Yang J, Gao W, Lane HC and Lempicki RA: DAVID: Database for annotation, visualization, and integrated discovery. Genome Biol 4: P3, 2003.

22. Szklarczyk D, Franceschini A, Kuhn M, Simonovic M, Roth A, Minguez P, Doerks T, Stark M, Muller J, Bork P, et al: The STRING database in 2011: Functional interaction networks of proteins, globally integrated and scored. Nucleic Acids Res 39 (Database Issue): D561-D568, 2011.

23. Shannon P, Markiel A, Ozier O, Baliga NS, Wang JT, Ramage D, Amin N, Schwikowski B and Ideker T: Cytoscape: A software environment for integrated models of biomolecular interaction networks. Genome Res 13: 2498-2504, 2003.

24. Bader GD and Hogue CW: An automated method for finding molecular complexes in large protein interaction networks. BMC Bioinformatics 4: 2, 2003.

25. Han H, Shim H, Shin D, Shim JE, Ko Y, Shin J, Kim H, Cho A, Kim E, Lee T, et al: TRRUST: A reference database of human transcriptional regulatory interactions. Sci Rep 5: 11432, 2015.

26. Livak KJ and Schmittgen TD: Analysis of relative gene expression data using real-time quantitative PCR and the 2(-Delta Delta C(T)) method. Methods 25: 402-408, 2001.

27. Norabuena E: Characterization of a human neuroblastoma cell line and its differentiation into dopamine neurons (unpublished $\mathrm{PhD}$ thesis). Mount Holyoke College, 2012.

28. Candito M, Thyss A, Albertini M, Deville A, Politano S, Mariani R and Chambon P: Methylated catecholamine metabolites for diagnosis of neuroblastoma. Med Pediatr Oncol 20: 215-220, 1992

29. Bozzi F, Luksch R, Collini P, Gambirasio F, Barzanò E, Polastri D, Podda M, Brando B and Fossati-Bellani F: Molecular detection of dopamine decarboxylase expression by means of reverse transcriptase and polymerase chain reaction in bone marrow and peripheral blood: Utility as a tumor marker for neuroblastoma. Diagn Mol Pathol 13: 135-143, 2004.

30. Lo Vasco VR: 1p36.32 rearrangements and the role of PI-PLC $\eta 2$ in nervous tumours. J Neurooncol 103: 409-416, 2011.

31. Zhou Y, Wing MR, Sondek J and Harden TK: Molecular cloning and characterization of PLC-eta2. Biochem J 391: 667-676, 2005

32. Modarressi MH, Taylor KE and Wolfe J: Cloning, characterization, and mapping of the gene encoding the human $\mathrm{G}$ protein gamma 2 subunit. Biochem Biophys Res Commun 272: 610-615, 2000.

33. Shuba YM, Teslenko VI, Savchenko AN and Pogorelaya NH: The effect of permeant ions on single calcium channel activation in mouse neuroblastoma cells: Ion-channel interaction. J Physiol 443: 25-44, 1991.

34. Barton AC and Sibley DR: Agonist-induced desensitization of D1-dopamine receptors linked to adenylyl cyclase activity in cultured NS20Y neuroblastoma cells. Mol Pharmacol 38 : 531-541, 1990

35. Fukunaga K and Miyamoto E: Role of MAP kinase in neurons. Mol Neurobiol 16: 79-95, 1998

36. Morón JA, Zakharova I, Ferrer JV, Merrill GA, Hope B, Lafer EM Lin ZC, Wang JB, Javitch JA, Galli A and Shippenberg TS: Mitogen-activated protein kinase regulates dopamine transporter surface expression and dopamine transport apacity. J Neurosci 23: 8480-8488, 2003.

37. Wagner EF and Nebreda AR: Signal integration by JNK and p38 MAPK pathways in cancer development. Nat Rev Cancer 9: 537-549, 2009.

38. Ries V, Silva RM, Oo TF, Cheng HC, Rzhetskaya M, Kholodilov N, Flavell RA, Kuan CY, Rakic P and Burke RE: JNK2 and JNK 3 combined are essential for apoptosis in dopamine neurons of the substantia nigra, but are not required for axon degeneration. J Neurochem 107: 1578-1588, 2008.
39. Herdman ML, Marcelo A, Ying H, Niles RM, Dhar S and Kiningham KK: Thimerosal Induces Apoptosis in a Neuroblastoma Model via the cJun N-terminal kinase pathway. Toxicol Sci 92: 246-253, 2006

40. Uribe V: The beta-tubulin gene TUBB2B is involved in a large spectrum of neuronal migration disorders. Clin Genet 77: 34-35, 2010.

41. Xu Y, Xu C, Kato A, Tempel W, Abreu JG, Bian C, Hu Y, Hu D, Zhao B, Cerovina T, et al: Tet3 CXXC domain and dioxygenase activity cooperatively regulate key genes for xenopus eye and neural development. Cell 151: 1200-1213, 2012.

42. Chang SH, Jung IS, Han GY, Kim NH, Kim HJ and Kim CW: Proteomic profiling of brain cortex tissues in a Tau transgenic mouse model of Alzheimer's disease. Biochem Biophys Res Commun 430: 670-675, 2013.

43. Pope WB, Lambert MP, Leypold B, Seupaul R, Sletten L, Krafft G and Klein WL: Microtubule-associated protein tau is hyperphosphorylated during mitosis in the human neuroblastoma cell line SH-SY5Y. Exp Neurol 126: 185-194, 1994.

44. Lorkowski S: RASL11B (RAS-like, family 11, member B). Atlas Genet Cytogenet Oncol Haematol 14: 747-750, 2010.

45. Stolle K, Schnoor M, Fuellen G, Spitzer M, Cullen P and Lorkowski S: Cloning, genomic organization, and tissue-specific expression of the RASL11B gene. Biochim Biophys Acta 1769: 514-524, 2007.

46. Ke XX, Zhang D, Zhao H, Hu R, Dong Z, Yang R, Zhu S, Xia Q, Ding HF and Cui H: Phox2B correlates with MYCN and is a prognostic marker for neuroblastoma development. Oncol Lett 9: 2507-2514, 2015

47. Bachetti T, Di Paolo D, Di Lascio S, Mirisola V, Brignole C, Bellotti M, Caffa I, Ferraris C, Fiore M, Fornasari D, et al: PHOX2B-mediated regulation of ALK expression: In vitro identification of a functional relationship between two genes involved in neuroblastoma. PLoS One 5: pii: e13108, 2010.

48. Desgrosellier JS and Cheresh DA: Integrins in cancer: Biological implications and therapeutic opportunities. Nat Rev Cancer 10: 9-22, 2010.

49. Judware R and Culp LA: Concomitant down-regulation of expression of integrin subunits by $\mathrm{N}$-myc in human neuroblastoma cells: Differential regulation of alpha2, alpha3 and beta1. Oncogene 14: 1341-1350, 1997.

50. Peng H, Ke XX, Hu R, Yang L, Cui H and Wei Y: Essential role of GATA3 in regulation of differentiation and cell proliferation in SK-N-SH neuroblastoma cells. Mol Med Rep 11: 881-886, 2015.

51. Fischer I, Shea TB, Sapirstein VS and Kosik KS: Expression and distribution of microtubule-associated protein 2 (MAP2) in neuroblastoma and primary neuronal cells. Brain Res 25: 99-109, 1986.

52. Braekeveldt N, Wigerup C, Gisselsson D, Mohlin S, Merselius M, Beckman S, Jonson T, Börjesson A, Backman T, Tadeo I, et al: Neuroblastoma patient-derived orthotopic xenografts retain metastatic patterns and geno- and phenotypes of patient tumours. Int J Cancer 136: E252-E261, 2015.

53. Brodeur GM: Neuroblastoma: Biological insights into a clinical enigma. Nat Rev Cancer 3: 203-216, 2003.

This work is licensed under a Creative Commons Attribution-NonCommercial-NoDerivatives 4.0 International (CC BY-NC-ND 4.0) License. 